\title{
TIME PERSPECTIVE AND PLACE OF RESIDENCE: AN EMPIRICAL STUDY
}

\author{
OKSANA M. SENYK \\ oksana.senyk85@gmail.com \\ Ivan Franko National University of Lviv, Ukraine
}

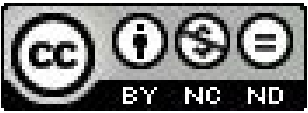

\begin{abstract}
The article focuses on relationship between time perspective and place of residence. Five hundred and eleven Ukrainian participants completed the Zimbardo Time Perspective Inventory [Ukrainian language version by Oksana Senyk (Senyk, 2012)] and a questionnaire that included the question about participants' current place of residence and their place of residence in childhood. Answers to the questionnaire made it possible to consider in the research respondents' change of residential place and its relation to the formation of different time orientations. The results have shown that the individual's time perspective is influenced not by the specific life situation represented by his past or current place of residence, but upon his whole life story that includes changing different residential settlements. The research has also indicated that among all types of settlements rural settlements most significantly contribute to the development of time orientation towards a positive past.
\end{abstract}

Key words: time perspective, time orientation, Zimbardo Time Perspective Inventory, place of residence.

\section{THE NOTION OF TIME PERSPECTIVE}

According to Professor Philip Zimbardo, time perspective is a fundamental value in constructing an individual's psychological time originating from cognitive processes that divide that individual's life experience into time frames of the past, present and future. The author defines time perspective as "a nonconscious process whereby the continual flows of personal and social experiences are assigned to temporal categories, or time frames, that help to give order, coherence, and meaning to those events" (Zimbardo, \& Boyd, 1999, p. 1271). These cognitive frames can reflect cyclic, repeated time patterns or unique, nonrecurring linear moments from an individual's life (Hall, 1999). They are used for encoding, storing and re-creating of experience as well as formulating expectations, goals, unexpected circumstances and imagined scenarios. Thus, time perspective penetrates into all spheres of human life and reflects personal tendency of the fact how subjective attitude to the past, present and future affects an individual's behaviour (Zimbardo, \& Boyd, 1999, 2010).

The very term "time perspective" has become widely used in scientific literature after the publication of a culturological and philosophical article by Lawrence Frank on this issue. The author characterizes time perspective as a dynamic basic property of human life accentuating interrelations and interactions of the past, 
present and future. According to L. Frank, an individual's time perspective can differ in various spheres of life and depends on norms and values of specific culture (Frank, 1939, p. 293-312).

The author of field theory Kurt Lewin includes time perspective into psychological life space of an individual and defines it as "the totality of the individual's views of his psychological future and psychological past existing at a given time" (Lewin, 2001, p. 263). These understandings belong to the group of factors of the present field and influence any behavioral act which is done in the present. Thus, time perspective looks like incorporating of the past and future, real and ideal life plan into the current moment plan (Lewin, 2001).

Later the theory of life space by K. Lewin was supported by Joseph Nuttin who focused on the perspective of the future considering it as a certain motivational formation that determines behaviour of the person in the present and depends upon his cognitive processes. By analogy with space perspective, time perspective consists of currently existing "mental perceptions" of events which in reality happen according to a certain time sequence with longer or shorter intervals between them. The distinguishing feature of time perspective lies in the fact that it originates not in real perception processes but in currently existing cognitive representations of events. According to J. Nuttin, "future and past events have an impact on present behavior to the extent that they are actually present on the cognitive level of behavioural functioning" (Nuttin, 2004, p. 356).

Being a dynamic formation, time perspective of an individual undergoes changes and transformations throughout his life. Starting from one's birth, time perspective extends and differentiation between real and unreal layers grows. A small child lives in the present, his time perspective includes only the nearest and the most immediate past and the nearest future. A child cannot draw a distinctive line between wishes and facts, hopes and expectations. During normal development of the child time linearity of his life space grows and his current behaviour is influenced by more distant past and future events. The process of socialization of individuals which lays the basis of one's norms and values, certain behavioural reactions and fundamentals of life perception, one's own perception and perception of other people goes through family institution or any other social institution which may replace it. The conditions under which socialization takes place significantly influence formation of his time perspective (Lewin, 2001; Mukhina, 1999). In other words, a model through which every culture and social environment builds their own image of the world will influence formation of a certain type of time perspective. For instance, orientation towards the future which is a necessary condition of success and in the Western culture is traditionally viewed as a precondition of psychological well-being, can signify naivety and absence of realism for other cultures: Stephen Bochner with colleague have found that children of Australian indigenous peoples, who prefer a small but immediate reward, have significantly higher IQ in comparison with their peers in every age group. In this culture smart children are taught not to rely on an uncertain future while the work done to achieve distant goals could mean lack of adaptability (see Nuttin, 2004, p. 379-380). 


\section{MEASURING TIME PERSPECTIVE}

The literary review by Joseph McGrath and Janice Kelly covers more than 200 different approaches to defining the concept of time perspective (McGrath, \& Kelly, 1986). The early attempts to measure it were mainly graphical, biographical and projective methods aimed at measuring various aspects of time perspective - time orientation, linearity, continuity, emotional mindset and balance, which complicated integration and processing of the findings. The first attempts to encompass the whole complexity of various aspects of time perspective in one instrument were made by Everett L. Shostrom (Time Competence Scale, Shostrom, 1964) and J. Nuttin (Time attitude Scale, Nuttin, 1985).

At the same time P. Zimbardo and Alex Gonzalez (Gonzalez, \& Zimbardo, 1985) offered a time perspective questionnaire which measured differences between present-oriented and future-oriented individuals. The instrument included separate time scales both within present and future frameworks, however, it showed an unstable factorial structure which resulted in further clarification and modification of scales to stabilize questionnaire factorial structure, increase in factorial load of separate points and growth of internal scale consistency. Orientation towards the past was added as well. As a result, five time factors with a high degree of reliability were obtained. The first factor - „Past Negative" - reflects a generally negative, aversive view of one's own past. Due to the reconstructive character of the past, such negative attitude could be conditioned both by the real experience of negative or traumatic moments and by negative reconstruction of not so complicated situations or by combination of these two variants. The second factor - "Present Hedonistic" - reflects a hedonistic, risk-taking attitude to one's own lifetime and presupposes enjoying the present moment irrespective of further consequences of one's behaviour. The third factor - „Future" - measures a general future orientation. The scale suggests that behaviour is dominated by effort-taking to achieve the goals set and possible rewards in the future. The fourth scale is „Past Positive”. Unlike the first factor that means trauma, pain and regret, this factor reflects warm and sentimental attitudes towards the past, when past experience and past times are recollected as something pleasant, "through rosecoloured spectacles $\square$ and nostalgia. The last, the fifth factor - „Present Fatalistic" - reveals a fatalistic, helpless, and hopeless attitude towards the future and life, individuals with such time orientation believe in fate and are certain that they cannot influence either present or future events of their life (Zimbardo, \& Boyd, 1999, p. 1274-1276). Besides measuring orientations towards different time zones - past, present and future - the scales also include emotional attitudes and specific behavioral strategies in respect of different time orientations.

Since publication of the questionnaire described (ZTPI - Zimbardo Time Perspective Inventory, 1999) there were no new instruments to measure time perspective in psychological literature. Owing to clear factorial structure, high level of reliability and validity ZTPI has become the leading questionnaire to determine time perspective and has been translated into more then twenty different languages. The Ukrainian adaptation of ZTPI was done by O. Senyk which resulted in 
reliable, internally consistent scales and confirmed the five-factor structure of the instrument which almost completely resembles factorial structure of the original (Senyk, 2012, p. 153-168). The whole adaptation procedure was agreed with the authors of the questionnaire and was done with their permission.

\section{RELATIONSHIP BETWEEN TIME PERSPECTIVE AND PLACE OF RESIDENCE}

P. Zimbardo states that geographical situation of a country is closely related to an individual's time perspective: the closer to the equator, the more expressed will be orientation towards the present and less towards the future. As an example, the author provides the differences in pace of life, time perspective and attitude to work of the population in the northern and southern part of Italy: the northern part of Italy harbors all its flourishing cities the residents of which are running business intensively, pay taxes on time and therefore, fill the country's Treasury. This is the area where main workforce is located who share their expressed orientation towards the future with their Austrian and German neighbours. On the contrary, the south of the country is characterized by lack of industrial enterprises and a high level of unemployment (about 50\%), which is explained by F. Zimbardo as a consequence of its residents' orientation towards the present: it is typical of such people to have low level of self-efficacy which is necessary to believe that something can be changed for the better (Zimbardo, \& Boyd, 2010, p. 107-108). Some researchers assume that time perspective is often related to the pace of life which varies not only in different countries but also in different inhabited settlements within one country (Levine, 1997). In particular, prevailing in big cities is, as a rule, orientation towards the future as there dominates the idea of achieving success, career prospects, earning big money, etc. At the same time, logically prevailing in towns and villages is orientation towards positive past as a reflection of keeping to family values and traditions. However, the studies by Anna Syrtsova, conducted in a Russian sample, did not show such differences in the profiles of time perspective of town residents and residents of multi-million cities. Vice versa, town residents have shown more expressed orientation towards the future, while representatives of multi-million cities were more oriented towards a hedonistic present. The author explains such findings by the entertainment industry which is available in big cities and contributes to the development of hedonistic attitudes toward one's present (Syrtsova, 2008).

Despite quite extensive research on relationship between time perspective and other psychological constructs (for instance, psychological well-being, personal characteristics, different types of abuses), not so much attention was paid to studying the dependence of its formation on the residential place of a person. The aim of this empirical research is to find out whether time perspective of an individual depends on the type and size of the residential settlement where a person spent his childhood and lives now. Another point of focus is what specific time orientations are influenced by the current residential place of a person and the place where a person lived in childhood. 


\section{RESEARCH PROCEDURE}

The research was conducted from June 2010 till March 2011. The participants of the research were 511 people aged from 16 to 72 years old $(M=21,8 ; S D=6,089)$, out of them 220 men and 291 women. The survey was done in group and individual forms and on a voluntary basis. One of the motivating factors to participate in the study was a guarantee to receive individual results either on paper or via email.

To measure time perspective of the participants the questionnaire ZTPI adapted into Ukrainian by O. Senyk (Senyk, 2012) was used. This questionnaire consists of 56 statements which make up five factors of ZTPI. These statements are to be rated, on a 5-point Likert scale, which makes it possible to measure the degree each feature is expressed.

To study the relationship between time perspective of the participants with their current residential place and residential place in childhood, the Q\&A form that was added to the questionnaire included questions about places where the participants spent their childhood and where they live now - whether in a big city (oblast centre), town, town-like settlement or village.

\section{RESULTS AND DISCUSSION}

To research differences between time orientations of people coming from different residential settlements the group of research participants, as based on the question „Where did you spend your childhood?", was divided into three subgroups: 1) those coming from oblast centres $(n=203), 2)$ those coming from towns $(n=134), 3)$ those coming from villages and town-like settlements $(n=173)$, which in total makes up 510 respondents (one participant did not give the answer to this question). People coming from villages and town-like settlements were enlisted into one subgroup as the number of participants who spent their childhood in town-like settlements was very small $(n=49)$, therefore, it was logical to combine this subgroup with the other one. As according to the legislation, a town-like settlement belongs to the same basic level of local government as a village (that means, has the same privileges concerning education, medicine, etc. and therefore, administratively it is closer to a village and not a town), the decision was taken to combine these two subgroups.

By means of the Scheffe test it was revealed that participants differ in terms of attitude to their past depending on the place where they spent their childhood: those who spent their childhood in a town-like settlement or village see their past in a more negative way as compared to those who spent it in towns and cities $(\mathrm{OblC}=2,68$, Town $=2,66$, Village $=2,85, \mathrm{~F}=4,71, \mathrm{p}=0,009$, Fig. 1$)$.

In a similar way based on the answers to the question "Where do you live now?" the respondents were also divided into three subgroups: 1$)$ residents of oblast centres $(n=386), 2)$ residents of towns $(n=55)$ and 3$)$ residents of villages and town-like settlements $(\mathrm{n}=70)$, in total -511 respondents. Comparison of these groups according to the method of Kruskal-Wallis has shown that residents of villages and town-like settlements are more positive about their past $(H=9,36$, $p=0,009$ ) as compared to the residents of oblast centres (Fig. 2). 


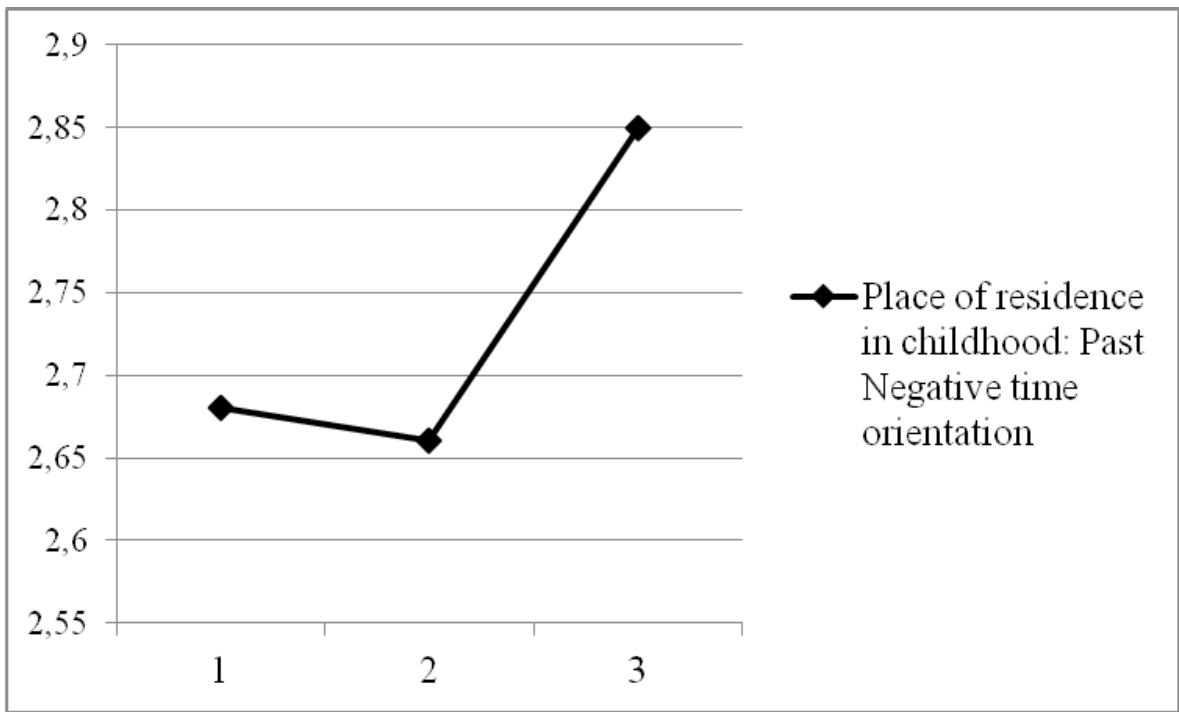

Fig. 1. Average values according to the scale "Past Negative" in subgroups divided on the basis of residential places in childhood: 1 - residents of oblast centres $(n=203), 2$ - town residents $(n=134), 3$ - residents of villages and town-like settlements $(\mathrm{n}=173)$.

Source: Prepared by author (own research).

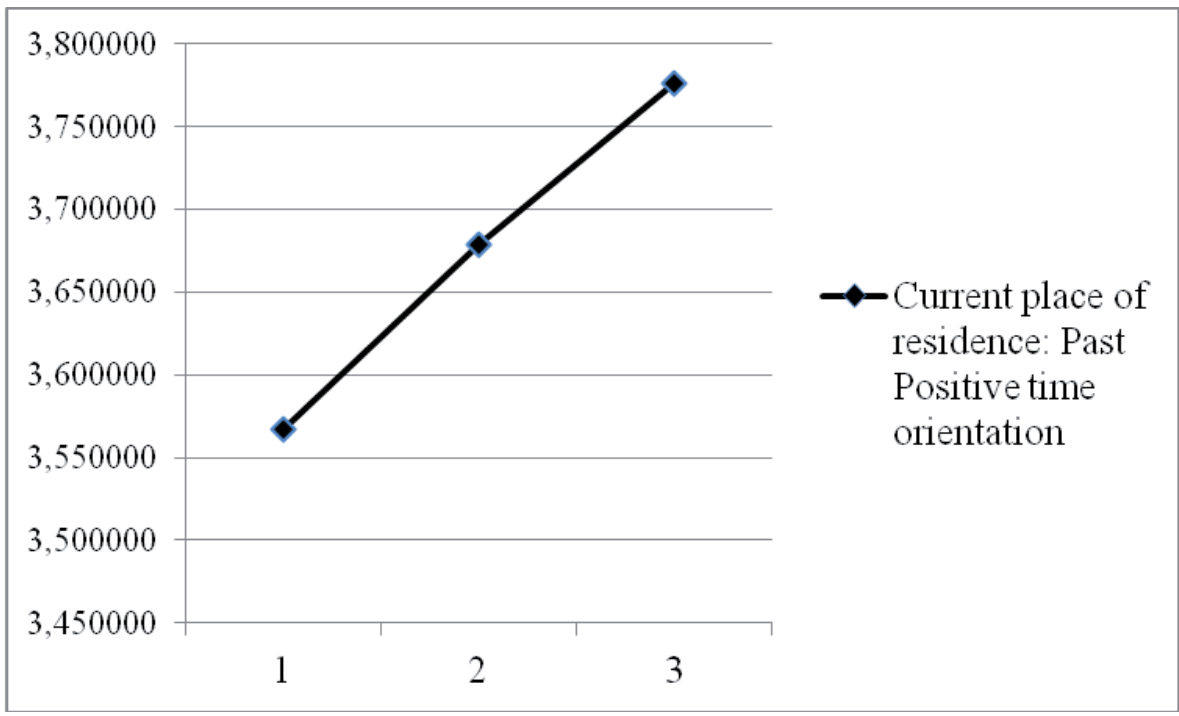

Fig. 2. Average values according to the scale "Past Positive" in subgroups divided on the basis of current residential places: 1 - residents of oblast centres $(n=386), 2$ town residents $(n=55), 3$ - residents of villages and town-like settlements $(n=70)$. Source: Prepared by author (own research). 
While planning this research we assumed that size and type of settlement where the respondents spent their childhood and where they currently live would be related to their time perspective. However, the results obtained were inconsistent: on the one hand, respondents who spent their childhood in villages or town-like settlements see their past in a more negative way in comparison with those coming from big cities. On the other hand, those respondents who currently live in a village or town-like settlement are more positive about their past than residents of oblast centres. But a large number of respondents did not change their residential places, therefore, it turns out that the same respondents (residents of villages and town-like settlements) are more negative and simultaneously more positive about their past in comparison with the other subgroup (residents of oblast centres).

It is also necessary to mention significant changes in the size of subgroups distinguished on the basis of current place of residence as compared to subgroups distinguished on the basis of childhood place of residence: the number of oblast centres residents has significantly grown, while the number of those who reside in villages and town-like settlements as well as small towns has decreased. It means that a lot of respondents have changed their place of residence, moreover, study of the history of respondents' residence places has demonstrated that this change happened in the direction of big cities, i.e. from a small town or village to the big city. Thus, the third subgroup distinguished on the basis of current residence place included only those respondents who spent their childhood in villages and town-like settlements and who live there up till now. However, in the first subgroup were those who spent all their life in oblast centres as well as those who moved to an oblast centre from a village or small town.

To solve contradictions that are revealed in time orientations towards negative and positive past with those coming from and residing in villages and big cities the decision was taken to have an additional subgroup based on the migration history of our respondents, namely: to distinguish one more subgroup among residents of oblast centres that will include those people who came there from a village or town-like settlement. Thus, we have got the following four subgroups of respondents: 1) residents of oblast centres including those who spent there their childhood as well as those who came to an oblast centre from a small town $(\mathrm{n}=278), 2)$ residents of small towns who had grown up there (n $=55), 3$ ) those respondents who spent their childhood and currently live in villages $(n=70), 4)$ residents of oblast centres who spent their childhood in a village or town-like settlement $(n=107)$. To compare these groups among themselves the method of Kruskall-Wallis was applied which resulted in statistically significant differences in respondents' time orientations towards past negative and past positive. The difference in expressing time orientation towards past positive was found among the first, third and fourth subgroups $(H=9,24, p=0,026)$ : individuals that spent their childhood in a village and currently reside there are more positive about their own past than those respondents who changed their residence place from the village to oblast centre as well as those who were born and are currently living in oblast centre (Fig 3). 


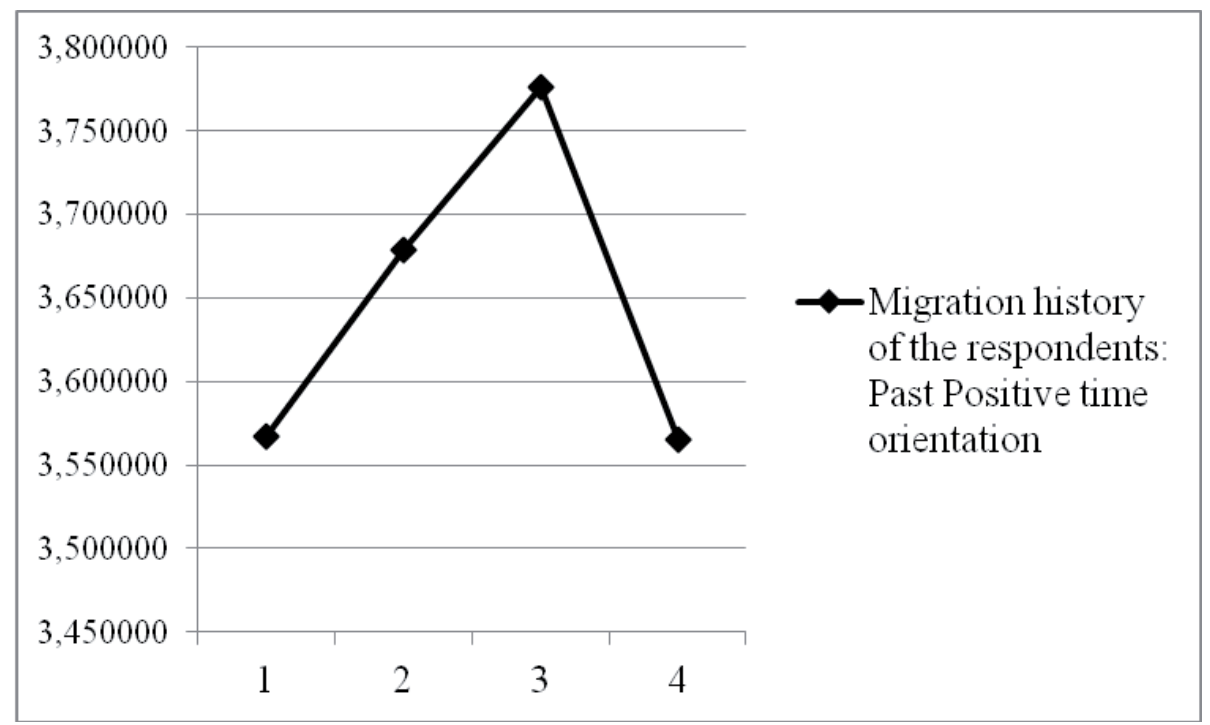

Fig. 3. Average values according to the scale „Past Positive” in subgroups divided on the basis of migration history of the respondents: 1 - residents of oblast centres including those who spent their childhood there as well as those who came to an oblast centre from a small town $(n=278), 2$ - residents of small towns who had grown up there $(\mathrm{n}=55), 3$ - those respondents who spent their childhood and currently live in villages $(n=70), 4$ - residents of oblast centres who spent their childhood in a village or town-like settlement $(n=107)$.

Source: Prepared by author (own research).

In contrast, individuals who spent their childhood in the village and later changed their residence place to an oblast centre are characterized by more expressed negative attitude toward their past as compared to those who have been living in oblast centres during whole their lives $(H=15,18, p=0,002$, Fig. 4).

The results obtained can explain the aforementioned contradictions. It is revealed that research participants who currently are living in settlements of the same type (village) but have different history of changing their residence place, have different time perspectives. We can assume that this was negative attitude to one's past, seeing it as something unpleasant that made the respondents from the fourth group change their residence place, search for new opportunities to improve their life as they viewed their life in the past as being negative. Those respondents who spent their past in the rural areas and are more positive about it, are not motivated to change something in their life, therefore, they remain residents of the rural areas. 


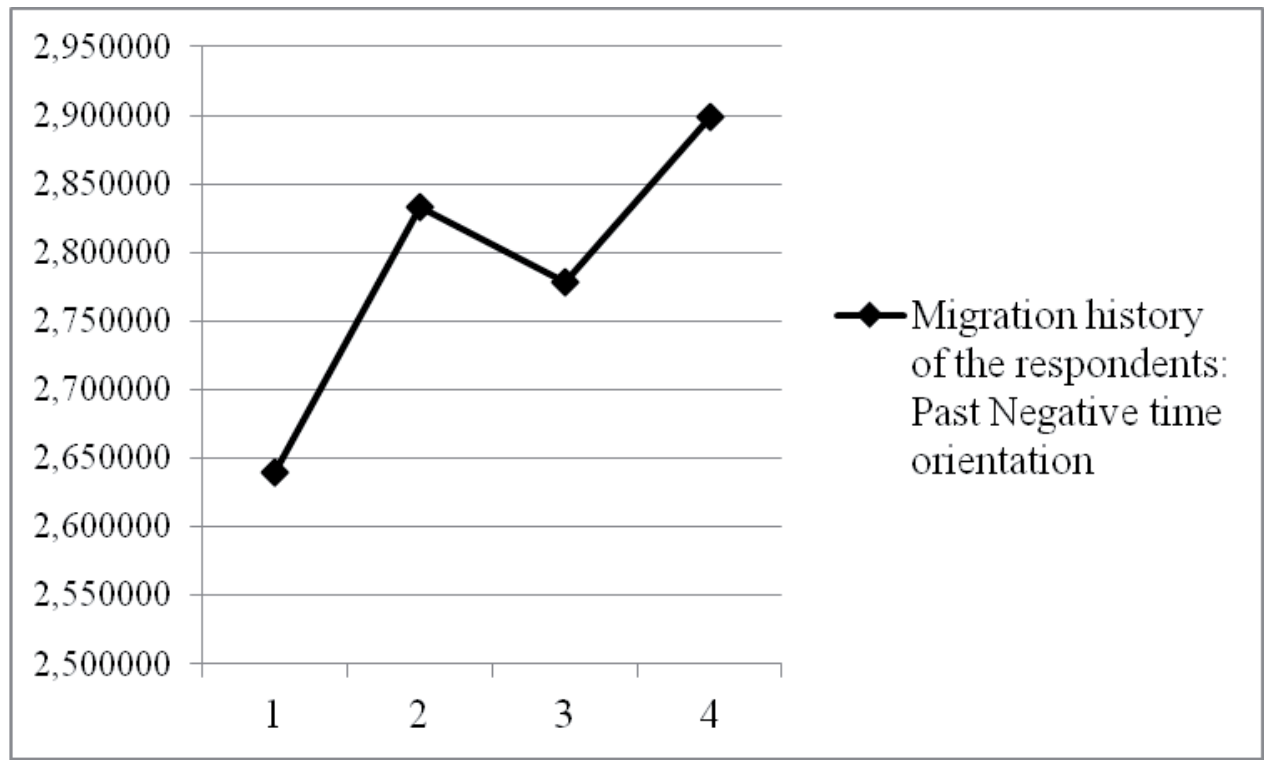

Fig. 4. Average values according to the scale "Past Negative” in subgroups divided on the basis of migration history of the respondents: 1 - residents of oblast centres including those who spent there their childhood as well as those who came to an oblast centre from a small town $(n=278), 2$ - residents of small towns who had grown up there $(n=55), 3$ - those respondents who spent their childhood and currently live in villages $(n=70), 4$ - residents of oblast centres who spent their childhood in a village or town-like settlement $(n=107)$.

Source: Prepared by author (own research).

According to P. Zimbardo, past positive time orientation makes an individual feel that his life is rooted, allows him to feel the connection with his identity and family, traditions and cultural heritage (Zimbardo, \& Boyd, 2010). One can assume that it is this feeling of grounded life that explains why these individuals do not change their residence place and remain residents of that settlement where they spent their childhood and youth. The very fact that more evident positive attitude towards one's past is characteristic of the villagers, in our opinion, can be explained by a way of life in different settlements as well as stronger orientation of village residents towards preserving traditions. Life in the village is more stable (due to certain cycles connected with household activities) and less dynamic which makes it possible to feel greater rootedness and contributes to the development of time orientation towards positive past. Keeping alive traditions - cultural, religious as well as traditions of everyday life - teaches an individual to love these traditions and through them - one's own past. Thus, owing to stability and preservation of traditions village residents develop more expressed past positive time orientation in comparison with representatives of other settlements. 
Those respondents who have less expressed past positive time orientation and who have more expressed negative attitude to their own past, have changed their residence place that can be explained by lack of rooted life and strong affection towards their past as well as by the aspirations to change their life for the better, by changing their residence place into a big city where there are more opportunities for jobs and self-realization.

It is also possible to assume that additional factors of more or less expressed positive attitude of the respondents towards their past is the result of solving cognitive dissonance. For instance, respondents who changed their residence place can unconsciously evaluate their own past in a less negative way to avoid regrets about this past and to justify their own choice. In contrast, those who have not changed their settlements can be more positive about their past to avoid regrets that they did not manage to change something in their life by changing their residence place. However, confirmation or rejection of this hypothesis requires more substantial and specific research.

\section{CONCLUSIONS}

The results obtained shed additional light on the issue of dependency of individual's time perspective on the size and type of his residential settlement. The study has found that certain facts from individual's past or present life, represented by his residential place in childhood and current residential place, do not determine his time perspective. However, the content of time perspective is influenced by the actual life situation of an individual that includes history of his life path. As our findings demonstrate, those who come from villages with different history of changing residential places have different time perspectives. In particular, they differ among themselves in expression of positive or negative attitude toward their own past: those who grew up and remained in rural areas have more expressed positive attitude toward their past as compared to those who spent their childhood in villages but later moved to big cities. However, individuals who changed their residential place from village to a big city have developed more pronounced past negative time orientation.

It is also important that those individuals who grew up and currently live in the village are the most positive about their past and in this attitude they differ from those respondents who grew up and currently live in a big city. This fact confirms our preliminary assumptions: a village with its settled way of life and orientation towards preservation of family values and traditions is the basis for more expressed past positive time orientation.

The prospects for future studies can be incorporation of the cultural context into the issue of dependence of time perspective on an individual's residential place.

\section{REFERENCES}

Frank, L.K. (1939). Time perspectives. Journal of Social Philosophy, 4, 293-312.

Gonzalez, A., \& Zimbardo, P.G. (1985). Time in perspective: A Psychology Today survey report. Psychology Today, May, 21-26. 
Hall, E. T. (1999). Taniec życia: inny wymiar czasu. Warszawa: Muza.

Levine, R. V. (1997). A Geography of Time: The Temporal Misadventures of a Social Psychologist. New York: Basic Books.

Lewin, K. Z. (2001). Dynamic Psychology: Selected Works. Moscow: Smysl.

McGrath, J., \& Kelly, J. (1986). Time and human interaction: Toward a social psychology of time. New York: Guildford Press.

Mukhina, V. S. (1999). Developmental Psychology: phenomenology of development, childhood, adolescence: textbook. Moscow: Academia.

Nuttin, J. M. (1985). Future time perspective and motivation. New Jersey: Hillsdale.

Nuttin, J. M. (2004). Motivation, Action and Future Time Perspective. Moscow: Smysl.

Senyk, O. M. (2012). Adaptation of Zimbardo Time Perspective Inventory (ZTPI). Social Psychology, 1-2 (51-52), 153-168.

Shostrom, E. L. (1964). An inventory for the measure of selfactualization. Educational and Psychological Measurement, 24, 207-218.

Syrtsova, A. (2008). Age-related Dynamics of Personality Time Perspective: PhD thesis in Psychology. Moscow: Moscow City Psychological-Pedagogical University.

Zimbardo, P. G., \& Boyd, J. N. (1999). Putting Time in Perspective: A Valid, Reliable Individual-Differences Metric. Journal of Personality and Social Psychology, 77 (6), 1271-1288.

Zimbardo Philip G., \& Boyd J. N. (2010). The Time Paradox: The New Psychology of Time That Will Change Your Life. Saint-Petersburg: Rech. 